\title{
An Improved Gas-Kinetic Scheme for Multimaterial Flows
}

\author{
Qibing Li* \\ AML, Department of Engineering Mechanics, Tsinghua University, Beijing 100084, \\ China.
}

Received 7 November 2018; Accepted (in revised version) 14 March 2019

\begin{abstract}
The efficiency of recently developed gas-kinetic scheme for multimaterial flows is increased through the adoption of a new iteration method in the kinetic nonmixing Riemann solver and an interface sharpening reconstruction method at a cell interface. The iteration method is used to determine the velocity of fluid interface, based on the force balance between both sides due to the incidence and bounce back of particles at the interface. An improved Aitken method is proposed with a simple hybrid of the modified Aitken method (Aitken-Chen) and the Steffensen method. Numerical tests validate its efficiency with significantly less calls to the function not only for the average number but also for the maximum. The new reconstruction is based on the tangent of hyperbola for interface capturing (THINC) but applied only to the volume fraction, which is very simple to be implemented under the stratified framework and capable of resolving fluid interface in mixture. Furthermore, the directional splitting is adopted rather than the previous quasi-one-dimensional method. Typical numerical tests, including several water-gas shock tube flows, and the shock-water cylinder interaction flow show that the improved gas-kinetic scheme can capture fluid interfaces much sharper, while preserving the advantages of the original one.
\end{abstract}

AMS subject classifications: 82B40, 76T10, 76M25

Key words: Gas-kinetic scheme, non-mixing interface model, stiffened equation of state, improved Aitken method, tangent of hyperbola for interface capturing.

\section{Introduction}

The compressible multimaterial flow is of great interest due to its importance in many engineering applications. A big challenge for a CFD method to capture the instability of fluid interface and the mixing of different fluids with the requirement of numerical dissipation, resolution, conservation and robustness, especially for high Mach number

${ }^{*}$ Corresponding author. Email address: lqb@tsinghua.edu. cn (Q. B. Li) 
and large density ratio [1,2]. Most existing studies are directly based on the macroscopic governing equations, such as the Euler equations, and the Riemann problem is usually solved to capture the field discontinuities for either a single fluid $[3,4]$ or across a fluid interface [5-7]. On the basis of the mesoscopic gas-kinetic theory, different numerical scheme can also be developed, which is suitable for not only continuum flows [8] but also rarefied flows [9-11]. For multimaterial flows, different kinetic Riemann solvers have been constructed such as those based on mass fractions of ideal gas mixtures [12-14], separated transport of ideal gas species [15-18] and stiffened gases [19,20], as well as the kinetic flux vector splitting for stiffened gas [21-24].

Recently, a new kinetic model has been proposed to solve the Riemann problem for stiffened gas interface [25]. In this model each gas is reflected back from the fluid interface which is moving with a velocity to achieve the force balance between both sides. It is second-order accurate in both space and time. Different from the previous kinetic Riemann solvers, the numerical mixing at the fluid interface is eliminated, which is dominant when the grid cell size is much larger than the width of physical interface. This numerical mixing may result in pressure oscillation near the material interface or contact discontinuity, especially when using local thermodynamic equilibrium assumption [26]. Furthermore, the extension of the kinetic model to high-order accuracy and multidimensional flows is straightforward. In addition, it is also simple to take into account the viscous effect inside each fluid component.

Based on the kinetic Riemann solver and with the help of the idea of discrete equation method [27] or stratified model [28,29] for fluid mixture, the gas-kinetic scheme for multimaterial flows (labeled as GKS-MMF) has been developed and shows good performance in many typical flows [25]. The advantages of the scheme include the conservation of each component, free of non-physical oscillations near fluid interface and the robustness in the flow with strong shock waves or large density ratio. The mesoscopic description of flow guarantees the inherent capability to characterize the complicated flow structures such as shock waves and other discontinuities. Even for inviscid flow, the corresponding numerical dissipation is more consistent and natural when compared with those based on macroscopic models.

To further increase the computational efficiency, two improvements are worthy of consideration. The first one is the iteration method in the kinetic Riemann solver to determine the interface velocity where the bisection method is applied. Although it works good but the convergence rate is slow which means more computational cost for the calculation of forces on both side of a fluid interface from the distribution function. Better alternative is required to reduce the iteration number, such as the Steffensen method. The second one is the reconstruction technique to sharpen the fluid interface. It is necessary for an interface capturing method where the numerical mixing can not be avoided especially based on a fluid mixture model. The tangent of hyperbola for interface capturing (THINC) technique is a good candidate which can effectively sharpen the interface in both incompressible [30] and compressible flows [31,32]. In the present study, the scheme is improved through these two modifications and is validated by typical tests, 
including several water-gas shock tube flows, and the interaction of a shock wave and a water cylinder.

\section{Brief description of GKS-MMF}

The previous proposed GKS-MMF [25] implements the homogeneous equilibrium mixture (HEM) model in which different fluid components achieve the dynamic and thermal equilibrium with equal temperature $T$, pressure $p$ and velocity $U=(U, V)^{T}$ within a computational cell during a time step. The stiffened equation of state for each component $s$ is considered,

$$
p=\rho^{(s)} R^{(s)} T-p_{c}^{(s)}, \quad R^{(s)}=C_{p}^{(s)}\left(\gamma^{(s)}-1\right) / \gamma^{(s)},
$$

where the parameters $\left(\gamma^{(s)}, C_{p}^{(s)}, p_{c}^{(s)}\right)$ are constants based on experimental data. $p_{c}$ represents the molecular attraction between molecules and it is zero for an ideal gas.

For a two-dimensional (2D) two-component flow, GKS-MMF updates the conservative variables using a finite volume formula,

$$
\boldsymbol{Q}_{i j}^{n+1}=\boldsymbol{Q}_{i j}^{n}+\frac{1}{S_{i j}} \sum_{m \in \partial S_{i j}} \Delta \boldsymbol{l}^{(m)} \cdot \int_{t^{n}}^{t^{n}+\Delta t} \boldsymbol{F}^{(m)} \mathrm{d} t,
$$

where $S_{i j}$ is the area of computational cell $(i, j)$ and $\Delta \boldsymbol{l}^{(m)}$ is the length vector of cell interface $m$. The conservative variables are

$$
Q=\alpha^{(1)} Q^{(1)}+\alpha^{(2)} Q^{(2)},
$$

where $\alpha^{(s)}, s=1,2$ is the volume fraction of component $s$, and $Q^{(s)}$ is the corresponding conservative variables,

$$
\begin{aligned}
& \boldsymbol{Q}^{(1)}=\left(\rho^{(1)}, 0, \rho^{(1)} \boldsymbol{U},(\rho E)^{(1)}\right)^{T}, \\
& \boldsymbol{Q}^{(2)}=\left(0, \rho^{(2)}, \rho^{(2)} \boldsymbol{U},(\rho E)^{(2)}\right)^{T}, \\
& (\rho E)^{(s)}=\frac{p+\gamma^{(s)} p_{c}^{(s)}}{\gamma^{(s)}-1}+\frac{\rho^{(s)}|\boldsymbol{U}|^{2}}{2} .
\end{aligned}
$$

Thus the total density is $\rho=\alpha^{(1)} \rho^{(1)}+\alpha^{(2)} \rho^{(2)}$. In addition, $\alpha^{(s)}$ satisfies the constraint

$$
\alpha^{(1)}+\alpha^{(2)}=1 \text {. }
$$

It should be noted that, for concision, in the conservative variables an additional component is included although it is zero, such as the second component in $Q^{(1)}$ and the first in $Q^{(2)}$. 


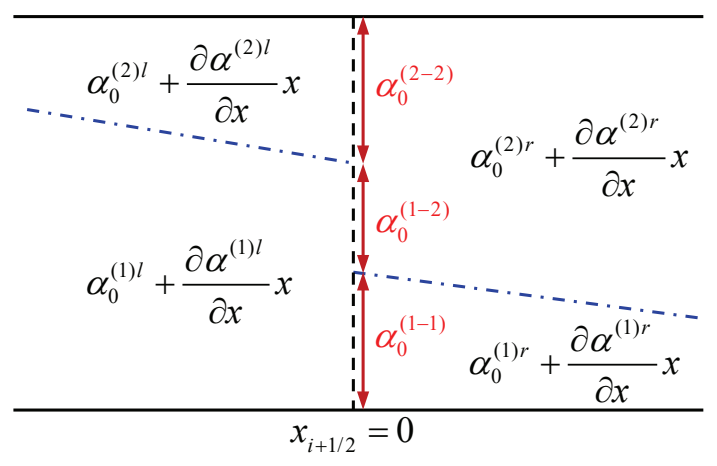

Figure 1: One-dimensional model to separate the two-component flow transport into three parts: the transport of each component and the interaction between different components, with reconstructed volume fraction $\alpha_{0}^{(1-1)}, \alpha_{0}^{(2-2)}$ and $\alpha_{0}^{(1-2)}$ at the cell interface, respectively. The subscript '0' of $\alpha$ indicates the location of cell interface $x_{i+1 / 2}=0$.

In GKS-MMF the numerical fluxes at a cell interface are computed from the solutions of a set of Riemann problems, formed through a similar idea of stratified model $[28,29]$ or discrete equation method [27]. As shown in Fig. 1 for a one-dimensional (1D) two-fluid flow these Riemann problems are corresponding to the transport of each component and the interactions between different components, with volume fractions $\alpha_{0}^{(1-1)}, \alpha_{0}^{(2-2)}$ and $\alpha_{0}^{(1-2)}$, respectively. Here the subscript ' 0 ' of $\alpha$ indicates the variable at cell interface $x_{i+1 / 2}=0$. They are determined by

$$
\begin{aligned}
& \alpha_{0}^{(1-1)}=\min \left(\alpha_{0}^{(1) l}, \alpha_{0}^{(1) r}\right), \\
& \alpha_{0}^{(2-2)}=\min \left(\alpha_{0}^{(2) l}, \alpha_{0}^{(2) r}\right), \\
& \alpha_{0}^{(1-2)}=\left|\Delta \alpha_{0}\right|, \quad \Delta \alpha_{0}=\alpha_{0}^{(1) r}-\alpha_{0}^{(1) l},
\end{aligned}
$$

where the superscripts ' $l$ ', ' $r$ ' represent the left and right sides of a cell interface, respectively. It can be easily verified that the constraint $\alpha_{0}^{(1-1)}+\alpha_{0}^{(2-2)}+\alpha_{0}^{(1-2)}=1$ is automatically satisfied.

Thus the total fluxes across cell interface $x_{i+1 / 2}=0$ can be computed by

$$
\boldsymbol{F}=\alpha_{0}^{(1-1)} \boldsymbol{F}^{(1-1)}+\alpha_{0}^{(2-2)} \boldsymbol{F}^{(2-2)}+\alpha_{0}^{(1-2)} \boldsymbol{F}^{(1-2)} .
$$

The fluxes for a single component $\boldsymbol{F}^{(1-1)}$ and $\boldsymbol{F}^{(2-2)}$ can be obtained through the usual Riemann solver for a single-component flow and $\boldsymbol{F}^{(1-2)}$ should be calculated with a Riemann solver for a fluid interface. In short, the interaction between different fluid components is taken into account through the Riemann problem for a fluid interface and the HEM assumption in the projection procedure of the finite volume scheme. Thus the key of GKS-MMF is to solve these two types of Riemann problem at a cell interface. 


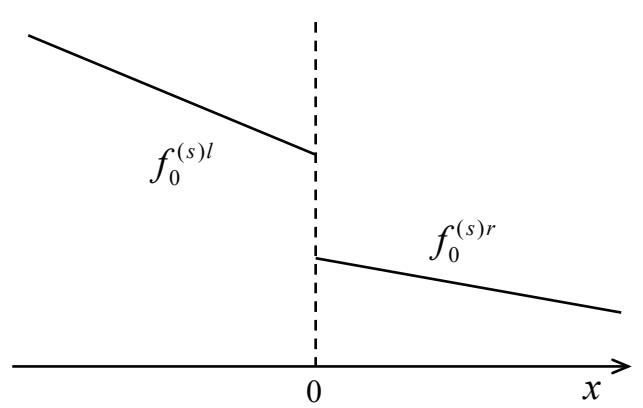

(a)

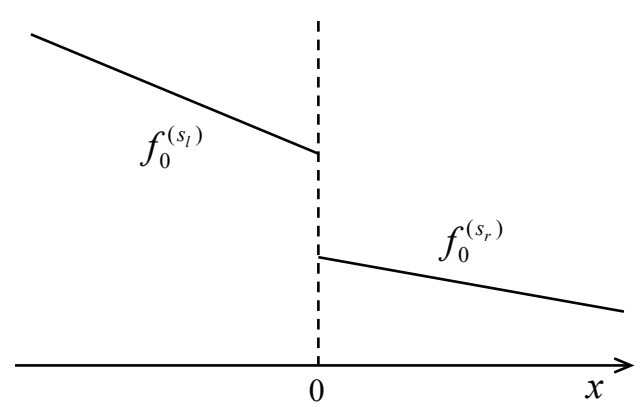

(b)

Figure 2: Riemann problem for single-component flow (a) and for flow interface (b) from piecewise linear initial distribution. The subscript ' 0 ' indicates the flow at initial time $t=0$.

Different from traditional scheme based on the macroscopic Euler equations, GKS solves [33] the Riemann problems through the distribution function $f^{(s)}$ for each component $s$, which is a function of space $x=(x, y)^{T}$, time $t$, particle velocity $\boldsymbol{u}=(u, v)^{T}$, and internal variable $\boldsymbol{\xi}^{(s)}$. As shown in Fig. $2, f_{0}^{(s)}$ is the initial distribution function coming from the reconstructed macroscopic conservative variables. In the Riemann problem for a two-component interface, the component index on both sides can be determined,

$$
s_{l}=1+\mathrm{H}\left[\Delta \alpha_{0}\right], \quad s_{r}=3-s_{l},
$$

in which $\mathrm{H}[x]$ is the Heaviside function.

\subsection{Gas-kinetic Riemann solver for single-component stiffened gas flow}

For a $2 \mathrm{D}$ single-component flow, the governing equation of distribution function $f^{(s)}$ is the BGK equation,

$$
\frac{\partial f^{(s)}}{\partial t}+\boldsymbol{u} \cdot \frac{\partial f^{(s)}}{\partial x}=\frac{g^{(s)}-f^{(s)}}{\tau^{(s)}}
$$

where $g^{(s)}$ is the equilibrium state approached by $f^{(s)}$. It is a Maxwellian distribution,

$$
g^{(s)}=\rho^{(s)}\left(\lambda^{(s)} / \pi\right)^{\left(K^{(s)}+2\right) / 2} \mathrm{e}^{-\lambda^{(s)}\left(\left|\boldsymbol{u}-\boldsymbol{U}^{(s)}\right|^{2}+\mathcal{\xi}^{(s) 2}\right)},
$$

where $\lambda^{(s)}$ is equal to $1 /\left(2 R^{(s)} T^{(s)}\right), R^{(s)}$ is the gas constant, and $T^{(s)}$ is the temperature. $\tau^{(s)}$ is the particle collision time. For a 2D flow, the total number of degrees of freedom $K^{(s)}$ in $\boldsymbol{\xi}^{(s)}$ is equal to $\left(5-3 \gamma^{(s)}\right) /\left(\gamma^{(s)}-1\right)+1$, where the particle motion in the $z$-direction is also included. The macroscopic conservative variables $Q^{(s)}$ and their fluxes along the 
$x_{m}$ direction, $\boldsymbol{F}_{m}^{(s)}$, can be determined by taking moments of the distribution function $f^{(s)}$

$$
\begin{aligned}
& Q^{(s)}=\int \psi^{(s)} f^{(s)} \mathrm{d} \Xi^{(s)}+\left(0,0,0,0, p_{c}^{(s)}\right)^{T}, \\
& \boldsymbol{F}_{m}^{(s)}=\int u_{m} \psi^{(s)} f^{(s)} \mathrm{d} \Xi^{(s)}+\left(0,0,-p_{c}^{(s)}, 0,0\right)^{T}, \quad m=1,2,
\end{aligned}
$$

where $\psi^{(s)}$ is the vector of moments

$$
\boldsymbol{\psi}^{(1)}=\left(1,0, \boldsymbol{u},\left(|\boldsymbol{u}|^{2}+\boldsymbol{\xi}^{(1) 2}\right) / 2\right)^{T}, \quad \boldsymbol{\psi}^{(2)}=\left(0,1, \boldsymbol{u},\left(|\boldsymbol{u}|^{2}+\boldsymbol{\xi}^{(2) 2}\right) / 2\right)^{T} .
$$

and $\mathrm{d} \Xi^{(s)}=\mathrm{d} u \mathrm{~d} \xi^{(s)}$ is the volume element in the phase space. The second term on the right side of Eqs. (2.9) and (2.10) comes from the pressure constant in the stiffened gas model which only affects the total energy and the normal momentum flux when compared with the ideal gas. In addition, one more component with zero value is employed in the moment vectors for concise writing, similar to the macroscopic conservative variables and fluxes.

BGK equation (2.8) has an integral solution,

$$
f^{(s)}\left(\boldsymbol{x}, t, \boldsymbol{u}, \boldsymbol{\xi}^{(s)}\right)=\frac{1}{\tau^{(s)}} \int_{0}^{t} g^{(s)}\left(\boldsymbol{x}^{\prime}, t^{\prime}, \boldsymbol{u}, \boldsymbol{\xi}^{(s)}\right) \mathrm{e}^{-\left(t-t^{\prime}\right) / \tau^{(s)}} \mathrm{d} t^{\prime}+\mathrm{e}^{-t / \tau^{(s)}} f_{0}^{(s)}\left(\boldsymbol{x}-\boldsymbol{u} t, \boldsymbol{u}, \boldsymbol{\xi}^{(s)}\right) .
$$

Here $x^{\prime}=x-u\left(t-t^{\prime}\right)$ is the trajectory of a particle motion and $f_{0}^{(s)}\left(x, u, \xi^{(s)}\right)$ is the initial gas distribution function at the beginning of each time step $\left(t=t^{n}=0\right)$. The local constant collision time can be calculated based on the local viscosity and pressure, $\tau^{(s)}=\mu^{(s)} / p^{(s)}$. Once $f_{0}^{(s)}$ and $g^{(s)}$ are known, the time-dependent distribution function can be obtained explicitly. For simplicity, the directional splitting method is adopted and the $x$ direction of the local coordinates is taken as an example in the following. $f_{0}^{(s)}$ can be constructed based on the first-order Chapman-Enskog expansion to approach the N-S equations and then the first-order Taylor expansion to achieve the second-order accuracy in space,

$$
\begin{aligned}
f_{0}^{(s)}\left(x, \boldsymbol{u}, \boldsymbol{\xi}^{(s)}\right)= & f_{0}^{(s) l}(1-\mathrm{H}[x])+f_{0}^{(s) r} \mathrm{H}[x] \\
= & \left(1+a^{(s) l} x-\tau^{(s)}\left(a^{(s) l} u+A^{(s) l}\right)\right)(1-\mathrm{H}[x]) g^{(s) l} \\
& +\left(1+a^{(s) r} x-\tau^{(s)}\left(a^{(s) r} u+A^{(s) r}\right)\right) \mathrm{H}[x] g^{(s) r},
\end{aligned}
$$

where $H[x]$ is used to account for the initial discontinuity. The equilibrium state $g^{(s)}$ is also constructed through the first-order Taylor expansion for the second-order accuracy,

$$
g^{(s)}\left(x, t, u, \boldsymbol{\zeta}^{(s)}\right)=\left(1+(1-\mathrm{H}[x]) \bar{a}^{(s) l} x+\mathrm{H}[x] \bar{a}^{(s) r} x+\bar{A}^{(s)} t\right) g_{0}^{(s)} .
$$

The terms $a^{(s) l}, a^{(s) r}, \bar{a}^{(s) l}, \bar{a}^{(s) r}, A^{(s) l}, A^{(s) r}$ and $\bar{A}^{(s)}$ take the similar form, $a^{(s) l}=a_{\chi}^{(s) l} \psi_{\chi}^{(s)}$, $\chi=1-5$, where all coefficients, $a_{\chi}^{(s) l}, \ldots, \bar{A}_{\chi}^{(s)}$, are local constants and determined uniquely from the spatial and temporal slopes of the reconstructed conservative variables $Q^{(s)}$. 
Thus the time evolution of the distribution function at the cell interface $x=0$ can be obtained through Eq. (2.11),

$$
\begin{aligned}
f^{(s)}\left(0, t, u, \xi^{(s)}\right)= & \left(1-C_{0}\right) g_{0}^{(s)}+\left(t-\tau+C_{1}\right) \bar{A}^{(s)} g_{0}^{(s)} \\
& +\left(-\tau^{(s)}+C_{1}+C_{2}\right)\left(\bar{a}^{(s) l} u \mathrm{H}[u]+\bar{a}^{(s) r} u(1-\mathrm{H}[u])\right) g_{0}^{(s)} \\
& +\left(C_{0}-\left(C_{1}+C_{2}\right) a^{(s) l} u-C_{1} A^{(s) l}\right) \mathrm{H}[u] g^{(s) l} \\
& +\left(C_{0}-\left(C_{1}+C_{2}\right) a^{(s) r} u-C_{1} A^{(s) r}\right)(1-\mathrm{H}[u]) g^{(s) r},
\end{aligned}
$$

where $C_{0}=\mathrm{e}^{-t / \tau^{(s)}}, C_{1}=\tau^{(s)} C_{0}$ and $C_{2}=t C_{0}$. It is a combination of Maxwellian distributions, thus the computational cost is comparable to traditional scheme based on macroscopic equations. Then the fluxes across the cell interface $\boldsymbol{F}^{(1-1)}$ and $\boldsymbol{F}^{(2-2)}$ in Eq. (2.6) can be calculated with Eq. (2.10). Details of the scheme can be found in references $[14,33]$.

\subsection{Gas-kinetic Riemann solver for stiffened gas interface}

A kinetic model was proposed for the Riemann problem with flow interface shown in Fig. 2(b) in the previous study [25]. It can eliminate the flow mixing at the interface which is different from the Riemann solver for single-component flows in which fluid particles can transport across the initial discontinuities. The incident fluid particles on both sides bounce back from the interface. Then the mean velocity $U_{\text {int }}$ of the interface within a time step can be determined by the constraint for normal stress (pressure) balance between both sides. Once $U_{\text {int }}$ is obtained, the density and other variables such as the fluxes can be obtained simultaneously.

The flow for each component is continuous, thus the distribution function of the incident particles near the left side of the interface $f_{\text {inc }}^{l}$ can be constructed through a similar procedure to Eq. (2.14),

$$
f_{\text {inc }}^{\left(s_{l}\right)}\left(t, u, v, \xi^{\left(s_{l}\right)}\right)=\left(1-\tau^{\left(s_{l}\right)}\left(a^{\left(s_{l}\right)} u+A^{\left(s_{l}\right)}\right)+t A^{\left(s_{l}\right)}\right) g_{0}^{\left(s_{l}\right)} .
$$

The initial equilibrium state $g_{0}^{\left(s_{l}\right)}$, the constants $a^{\left(s_{l}\right)}$ and $A^{\left(s_{l}\right)}$ are determined by the reconstructed initial macroscopic variables $Q_{0}^{\left(s_{l}\right)}$ and their spatial slopes $\partial Q_{0}^{\left(s_{l}\right)} / \partial x$, respectively.

For simplicity the distribution function of the reflecting particles is obtained through the specific reflecting model,

$$
f_{\text {ref }}^{\left(s_{l}\right)}\left(t, u, v, \xi^{\left(s_{l}\right)}\right)=f_{\text {inc }}^{\left(s_{l}\right)}\left(t, 2 U_{\text {int }}-u, v, \xi^{\left(s_{l}\right)}\right)
$$

Thus the final gas distribution function on the left side of the interface can be written as

$$
f^{\left(s_{l}\right)}\left(t, u, v, \boldsymbol{\xi}^{\left(s_{l}\right)}\right)=\left.f_{\text {inc }}^{\left(s_{l}\right)}\left(t, u, v, \boldsymbol{\xi}^{\left(s_{l}\right)}\right)\right|_{u>U_{\text {int }}}+\left.f_{\text {ref }}^{\left(s_{l}\right)}\left(t, u, v, \boldsymbol{\xi}^{\left(s_{l}\right)}\right)\right|_{u<U_{\text {int }}} .
$$


The averaged normal stress within a time step $\Delta t$ on the left side of the interface is then computed through the integration of the above distribution function, which can be simplified according to the symmetry about $u=U_{\text {int, }}$,

$$
\bar{P}_{11}^{l}=\frac{2}{\Delta t} \int_{0}^{\Delta t} \int_{u>U_{\text {int }}}\left(u-U_{\text {int }}\right)^{2} f_{\text {inc }}^{\left(s_{l}\right)}\left(t, u, v, \xi^{\left(s_{l}\right)}\right) \mathrm{d} \Xi^{\left(s_{l}\right)} \mathrm{d} t-p_{c}^{\left(s_{l}\right)} .
$$

Similarly, the normal stress on the right side of the interface can be computed,

$$
\bar{P}_{11}^{r}=\frac{2}{\Delta t} \int_{0}^{\Delta t} \int_{u<U_{\text {int }}}\left(u-U_{\text {int }}\right)^{2} f_{\text {inc }}^{\left(s_{r}\right)}\left(t, u, v, \xi^{\left(s_{r}\right)}\right) \mathrm{d} \Xi^{\left(s_{r}\right)} \mathrm{d} t-p_{c}^{\left(s_{r}\right)} .
$$

Now the only unknown is the interface velocity $U_{\text {int }}$ which can be determined uniquely by the constraint of force balance,

$$
\bar{P}_{11}^{l}\left[U_{\text {int }}\right]-\bar{P}_{11}^{r}\left[U_{\text {int }}\right]=0 .
$$

An iteration method such as the bisection method is required to solve the above nonlinear algebraic equation.

After $U_{\text {int }}$ is determined, the last fluxes in Eq. (2.6) can be computed,

$$
\begin{aligned}
\boldsymbol{F}^{(1-2)}= & (1-\mathrm{H}[\zeta]) \int u \psi^{\left(s_{l}\right)} f^{\left(s_{l}\right)} \mathrm{d} \Xi^{\left(s_{l}\right)}+\mathrm{H}[\zeta] \int u \psi^{\left(s_{r}\right)} f^{\left(s_{r}\right)} \mathrm{d} \Xi^{\left(s_{r}\right)} \\
& +\left(0,0,-(1-\mathrm{H}[\zeta]) p_{c}^{\left(s_{l}\right)}-\mathrm{H}[\zeta] p_{c}^{\left(s_{r}\right)}, 0,0\right)^{T} .
\end{aligned}
$$

Here the variable $\zeta=\Delta \alpha_{0} U_{\text {int }}$ is used to indicate which component is moving across the cell interface. To reduce the computational cost, when $\Delta \alpha_{0}<5 \epsilon$ the Riemann solver is replaced by a simple upwind scheme to calculate the fluxes $\boldsymbol{F}^{(1-2)}$. Here the small value $\epsilon=10^{-5} \sim 10^{-7}$ is simply chosen in the mixture model to represent the void fraction of one fluid inside the other 'pure' component in the initial flow fields. Numerical experiments show that the simulated results are not sensitive to $\epsilon$ when it is less than about $10^{-4}$.

It should be mentioned that it is simple to implement the present GKS-MMF because the subroutines of GKS for a single-component flow [14] can be directly adopted in the code for the above-mentioned two Riemann solvers. For the stiffened gas, only an additional modification of the normal stress is required, that is, to minus the pressure constant $p_{c}$ [19]. Additionally, the updated and stored quantities are the conservative variables $Q$, thus the volume fraction $\alpha^{(s)}$ should be computed from the components of $Q$ by solving a quadratic equation. Then other variables such as pressure $p$ and temperature $T$ can be determined. Details can be found in the previous study [25].

\section{Acceleration of the iteration with an improved Aitken method}

In the previous study, a simple bisection method is adopted to calculate the interface velocity $U_{\text {int }}$. Considering the computational cost to calculate the normal stress through 
Eq. (2.20), it is worth adopting a better iteration method to reduce the number of iteration. The Steffensen method is tried which has much better convergence rate than the bisection method and is free of additional slope computations for the equation to be solved when compared with the Newton method.

To use the Steffensen method, Eq. (2.20) is rewritten as

$$
U_{\text {int }}=\mathcal{F}\left[U_{\text {int }}\right] \triangleq U_{\text {int }}+\frac{\bar{P}_{11}^{l}\left[U_{\text {int }}\right]-\bar{P}_{11}^{r}\left[U_{\text {int }}\right]}{M},
$$

where the variable $M=\rho^{l} c^{l}+\rho^{r} c^{r}$ is determined by the density and sound speed on both sides. The Steffensen iteration function is

$$
\begin{aligned}
& U_{\text {int }}^{*}=\mathcal{F}\left[U_{\text {int }}^{(k)}\right], \quad U_{\text {int }}^{* *}=\mathcal{F}\left[U_{\text {int }}^{*}\right], \\
& U_{\text {int }}^{(k+1)}=U_{\text {int }}^{(k)}-\frac{\left(U_{\text {int }}^{*}-U_{\text {int }}^{(k)}\right)^{2}}{U_{\text {int }}^{* *}-2 U_{\text {int }}^{*}+U_{\text {int }}^{(k)}}, \quad k=0,1,2, \cdots .
\end{aligned}
$$

The convergence criteria is

$$
\min \left(\left|U_{\text {int }}^{*}-U_{\text {int }}^{k}\right|,\left|U_{\text {int }}^{* *}-U_{\text {int }}^{*}\right|,\left|U_{\text {int }}^{(k+1)}-U_{\text {int }}^{(k)}\right|\right)<\hat{\varepsilon} / M,
$$

where $\hat{\varepsilon}$ is a small value. Unless otherwise specified $\hat{\varepsilon}=10^{-8} \min \left(p^{l}, p^{r}\right)$ is chosen in the present study. The initial value for the iteration is chosen as

$$
U_{\text {int }}^{(0)}=\left(\rho^{l} U^{l}+\rho^{r} U^{r}\right) /\left(\rho^{l}+\rho^{r}\right) .
$$

In the Steffensen method, there are two calls to the function $\mathcal{F}$ in each iteration. To reduce the number of calls, a modified Aitken method, called Aitken-Chen method is also tried [34],

$$
U_{\text {int }}^{(k+1)}=U_{\text {int }}^{(k)}-\frac{\left(U_{\text {int }}^{(k)}-U_{\text {int }}^{(k-1)}\right)\left(\mathcal{F}\left[U_{\text {int }}^{(k)}\right]-U_{\text {int }}^{(k)}\right)}{\mathcal{F}\left[U_{\text {int }}^{(k)}\right]-\mathcal{F}\left[U_{\text {int }}^{(k-1)}\right]+U_{\text {int }}^{(k-1)}-U_{\text {int }}^{(k)}}, \quad k=0,1,2, \cdots .
$$

In this method the values at the previous step $U_{\text {int }}^{(k-1)}$ and $\mathcal{F}\left[U_{\text {int }}^{(k-1)}\right]$ are used in the slope approximation of the Aitken method, thus only one call to the function is required for each iteration. The convergence criteria is

$$
\min \left(\left|\mathcal{F}\left[U_{\text {int }}^{(k)}\right]-U_{\text {int }}^{k}\right|,\left|U_{\text {int }}^{(k+1)}-U_{\text {int }}^{(k)}\right|\right)<\hat{\varepsilon} / M .
$$

This method works well in the present GKS-MMF, except that for some interface cells, it can hardly converge. Thus a hybrid method is adopted. The Aitken-Chen method is implemented at first. If it does not converge after 4 iterations, then the Steffensen method is tried, starting with the initial value from the Aitken-Chen method. It is labeled as improved Aitken method here. Numerical tests show a successful reduction of both average and maximum numbers of calls to the function $\mathcal{F}$. 


\section{Hyperbolic tangent reconstruction for volume fraction}

The second improvement is the adoption of the idea of THINC $[30,31]$ to sharpen the fluid interface. In the present gas-kinetic scheme for compressible flows, it is only used for the reconstruction of the volume fraction, similar to the treatment of Nonomura et al. [32]. It works well for non-mixing flows. However, for mixture flows or near the thick interface due to numerical mixing, oscillation can be observed. Thus the upper and lower bounds are determined by the local neighboring cells [31], rather than two limiting values ' 0 ' and ' 1 '. For simplicity the $1 \mathrm{D}$ reconstruction is implemented under the directional splitting framework.

To reconstruct the volume fraction in a computational cell $i$, the first step is to identify whether it is inside a fluid interface, based on the cell averaged volume fraction $\alpha_{i}$. The assessment criterion is

$$
\varepsilon<\alpha_{i}<1-\varepsilon, \quad\left(\alpha_{i+1}-\alpha_{i}\right)\left(\alpha_{i}-\alpha_{i-1}\right)>0,
$$

where $\varepsilon$ is a small positive parameter.

If $\alpha_{i}$ satisfies the above criterion, then the hyperbolic tangent reconstruction is implemented,

$$
\begin{aligned}
& \alpha(x)=\alpha_{\min }+\frac{\Delta \alpha}{2}\left(1+\sigma_{i} \tanh \left[\beta\left(\frac{x-x_{i-1 / 2}}{\Delta x_{i}}-\tilde{x}_{i}\right)\right]\right), \\
& \sigma_{i}=\operatorname{sign}\left[\alpha_{i+1}-\alpha_{i-1}\right], \quad x \in\left[x_{i-1 / 2}, x_{i+1 / 2}\right], \quad \Delta x_{i}=x_{i+1 / 2}-x_{i-1 / 2} .
\end{aligned}
$$

Here $\beta$ is a constant to control the thickness of the volume fraction jump $\Delta \alpha=\alpha_{\max }-\alpha_{\min }$ and set as $\beta=2.3$ in the present study. The bounds of the jump are determined from the neighboring cells,

$$
\alpha_{\min }=\min \left[\alpha_{i-3 / 2}^{r}, \alpha_{i+3 / 2}^{l}\right], \quad \alpha_{\max }=\max \left[\alpha_{i-3 / 2}^{r}, \alpha_{i+3 / 2}^{l}\right],
$$

where $\alpha_{i-3 / 2}^{r}$ is the volume fractions at the left boundary of cell $i-1$ and $\alpha_{i+3 / 2}^{l}$ the right boundary of cell $i+1$. They are computed through a usual reconstruction, such as using the van Leer limiter. Thus the only unknown is the center location $\tilde{x}_{i}$ which can be determined by the conservation of volume fraction,

$$
\frac{1}{\Delta x_{i}} \int_{x_{i-1 / 2}}^{x_{i+1 / 2}} \alpha(x) \mathrm{d} x=\alpha_{i} \text {. }
$$

It gives the value

$$
\tilde{x}_{i}=\ln \left[\left(D_{1} D_{2}-1\right) /\left(1-D_{1} / D_{2}\right)\right] /(2 \beta), \quad D_{1}=\mathrm{e}^{\left(1-2 \alpha_{i}\right) \beta / \sigma_{i}}, \quad D_{2}=\mathrm{e}^{\beta} .
$$

Thus the volume fraction at the cell interface can be obtained using Eq. (4.2),

$$
\begin{aligned}
\alpha_{i-1 / 2}^{r} & =\alpha_{\min }+(\Delta \alpha / 2)\left(1+\sigma_{i} \tanh \left[-\beta \tilde{x}_{i}\right]\right), \\
\alpha_{i+1 / 2}^{l} & =\alpha_{\min }+(\Delta \alpha / 2)\left(1+\sigma_{i} \tanh \left[\beta\left(1-\tilde{x}_{i}\right)\right]\right) .
\end{aligned}
$$


This hyperbolic tangent reconstruction for volume fraction labeled as 'TH0' can dramatically reduce the thickness of fluid interface. However a very small early variation may be observed near the upstream of a fluid interface. An improvement is adopted through a simple hybrid method,

$$
\alpha_{i+1 / 2}=\omega \alpha_{j+1 / 2}^{\mathrm{TH} 0}+(1-\omega) \alpha_{j+1 / 2}^{\mathrm{N}},
$$

where $\alpha_{i+1 / 2}^{\mathrm{TH} 0}$ is computed through the above-mentioned hyperbolic tangent reconstruction and $\alpha_{i+1 / 2}^{\mathrm{N}}$ from the usual reconstruction such as with the van Leer limiter. The weighting factor $\omega$ is chosen as

$$
\begin{aligned}
& \omega= \begin{cases}\left(1+\tanh \left[\left(\alpha_{i}-\left(\alpha_{\min }+\varepsilon_{c}\right)\right) / \Delta \varepsilon\right]\right) / 2, & \alpha_{i}<\left(\alpha_{\min }+\alpha_{\max }\right) / 2, \\
\left(1-\tanh \left[\left(\alpha_{i}-\left(\alpha_{\max }-\varepsilon_{c}\right)\right) / \Delta \varepsilon\right]\right) / 2, & \text { else, }\end{cases} \\
& \Delta \varepsilon=\varepsilon_{2}-\varepsilon_{1}, \quad \varepsilon_{c}=\left(\varepsilon_{1}+\varepsilon_{2}\right) / 2 .
\end{aligned}
$$

The hybrid bounds are simply set as $\varepsilon_{1}=2 \varepsilon, \varepsilon_{2}=8 \varepsilon$ where the small value $\varepsilon$ is the same as in Eq. (4.1). Numerical tests show that the results are not sensitive to the value of $\varepsilon_{1}, \varepsilon_{2}$, and even $\varepsilon$. This hybrid reconstruction is labeled as ' $\mathrm{TH}$ '.

The above-mentioned interface sharpening method is simple and effective. Noting that it only requires the manipulation of volume fraction, this is equivalent to modify the portion of numerical fluxes from different interaction in Eq. (2.6) under the stratified framework. Thus the advantages of original GKS-MMF can be well preserved, such as the conservation, no non-physical oscillations near fluid interface, and robustness in the multimaterial flow with strong shock waves or large density ratio.

\section{Numerical tests}

Several typical gas-water flows are simulated to validate the present improved GKSMMF, including the 1D shock tube problems with different initial pressure and volume fraction jumps, and the shock-water cylinder interaction. Unless otherwise stated, in these numerical examples all variables are presented using SI units. The constants for air are

$$
\left(\gamma_{g}, R_{g}, p_{c}\right)=(1.4,288,0)
$$

and

$$
\left(\gamma_{w}, C_{p w}, p_{c}\right)=\left(1.9276,8076.6,1.1373 \times 10^{9}\right)
$$

for water. For simplicity the volume fraction of fluid $1, \alpha^{(1)}$ is written as $\alpha$. For shock tube problems, the computational domain is $[0,10]$ divided by $N=200$ uniform cells. The initial discontinuity is located at $x=5$. The small values $\epsilon=10^{-5}$ and $\varepsilon=2 \epsilon$ are chosen in all tests. The CFL number is set to 0.6. These cases are inviscid and the collision time is calculated by

$$
\tau=C_{\tau 1} \Delta t+C_{\tau 2} \Delta t\left|p^{l}-p^{r}\right| /\left(p^{l}+p^{r}\right)
$$


where $p^{l}, p^{r}$ are the reconstructed pressure at two sides of a cell interface. The values of constants $C_{\tau 1}, C_{\tau 2}$ can be set between 0 and 1 . In the present work, $C_{\tau 1}=0.1$ and $C_{\tau 2}=0.2$ are chosen and the computation is not sensitive to them. For $2 \mathrm{D}$ cases, the code is parallelized with MPI (message passing interface) [35].

\subsection{Moving contact discontinuity problem}

The first shock tube flow is considered to validate the capability to capture a pure fluid interface. The initial field is set as [29]

$$
(\alpha, U, p, T)^{l}=\left(1-\epsilon, 100,10^{5}, 300\right), \quad(\alpha, U, p, T)^{r}=\left(\epsilon, 100,10^{5}, 300\right) .
$$

The corresponding analytical solution is a moving contact discontinuity with constant velocity and the pressure is uniform throughout the flow field. Fig. 3 shows the results

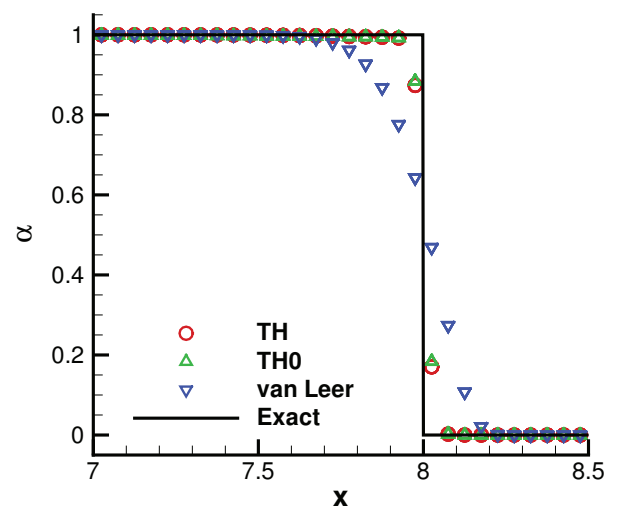

(a)

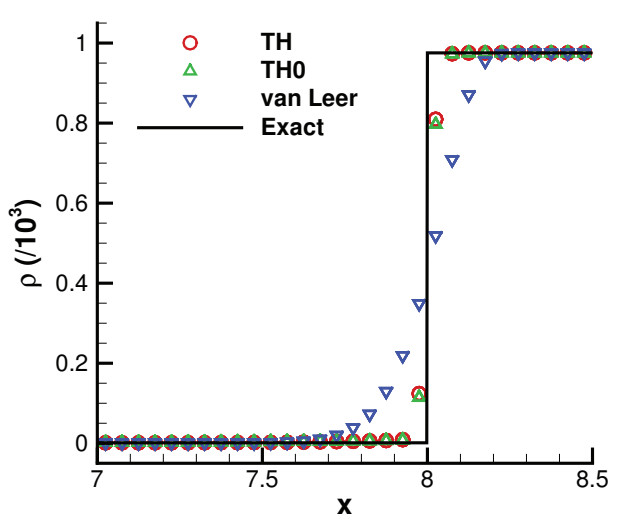

(c)

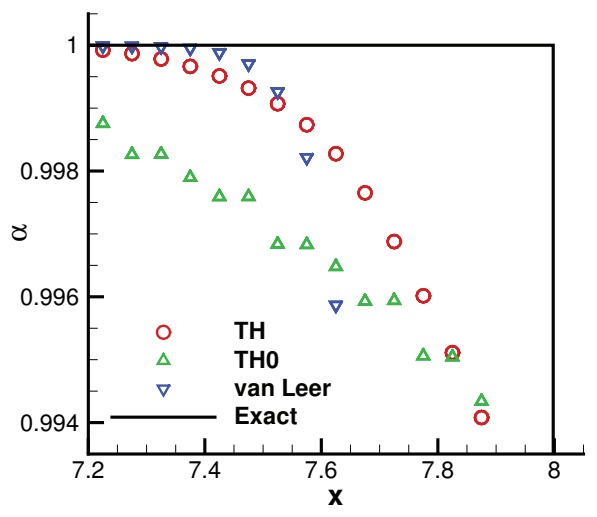

(b)

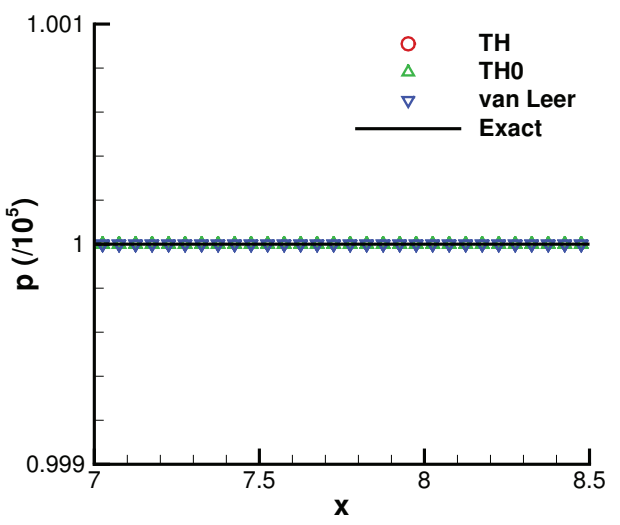

(d)

Figure 3: Profiles of air volume fraction and the enlarged view (a, b), density (c) and pressure (d) for air-water moving contact discontinuity problem. 
at time $t=0.03$ computed by the present GKS-MMF with different reconstruction of the volume fraction. The contact discontinuity is well predicted and no oscillation can be observed. The relative variation across the interface is at the magnitude of $10^{-8}$ for pressure and $10^{-10}$ for velocity, respectively. In addition, the positivity of volume fraction is preserved during the simulation.

With the help of THINC technique labeled by ' $\mathrm{TH}^{\prime}$ ' and ' $\mathrm{THO}$ ', the predicted fluid interface is much sharper, with only three cells inside the interface, while about nine cells is required when using the van Leer limiter [25]. Thus the resolution of fluid interface is dramatically enhanced. Furthermore, in the volume fraction predicted by the original THINC reconstruction ( $\mathrm{THO}$ ) a very small early variation can be observed near the upstream of fluid interface, as shown in Fig. 3(b). Through the hybrid of THINC and the van Leer limiter, this flaw is effectively remedied.

\subsection{Air-to-water shock tube problem}

This flow contains a strong initial discontinuity with large pressure ratio of $10^{4}$ where the gas pressure is higher than that in water [29]:

$$
(\alpha, U, p, T)^{l}=\left(1-\epsilon, 0,10^{9}, 300\right), \quad(\alpha, U, p, T)^{r}=\left(\epsilon, 0,10^{5}, 300\right) .
$$

As shown in Fig. 4, the fluid interface, the very strong shock in water and the rarefaction wave in air are well captured, which is in good agreement with the analytical solution and numerical study using the stratified model and $\mathrm{AUSM}^{+}$-up scheme [29]. Again, no non-physical oscillation can be found not only near the fluid interface, but also around the strong shock waves. When compared with the previous study [25], the present predicted fluid interface is obviously sharper, which can be clearly observed in the volume fraction and density fields. The THINC reconstruction works well in this challenging flow with large density ratio and pressure ratio across the initial discontinuity. It is interesting that the temperature across the interface seems not to change much, when compared with the result predicted without THINC. Further investigation is required.

\subsection{Water-air mixture shock tube problem}

To the present GKS-MMF based on mixture model, the performance in mixture flow is of great important. In fact, the fluid interface is also like a mixture, as it is usually across several computational cells. Thus the shock tube problem in water-gas mixture [36] is simulated. The constants for water are set as $\left(\gamma_{w}, C_{p w}, p_{c}\right)=\left(2.8,4186,8.5 \times 10^{8}\right)$ for better comparison with previous studies $[19,25]$. The initial flow field is given by

$$
(\alpha, U, p, T)^{l}=\left(0.25,0,2 \times 10^{7}, 308.15\right), \quad(\alpha, U, p, T)^{r}=\left(0.1,0,10^{7}, 308.15\right) .
$$

Fig. 5 shows the predicted expansion wave, shock wave and the contact discontinuity, which are in good agreement with the previous studies using a mixing gas-kinetic model 


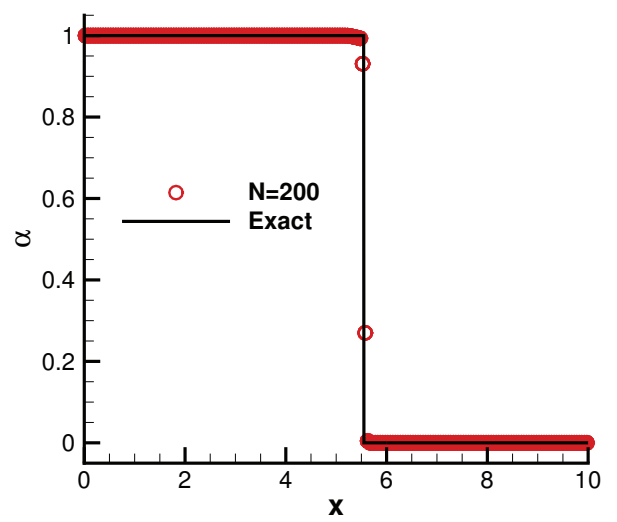

(a)

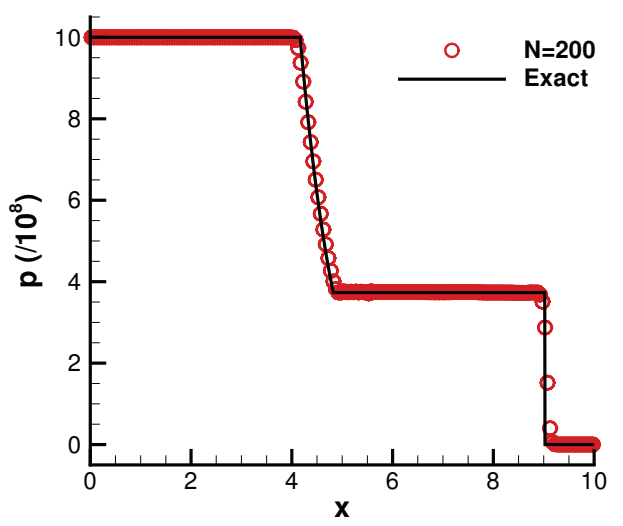

(c)

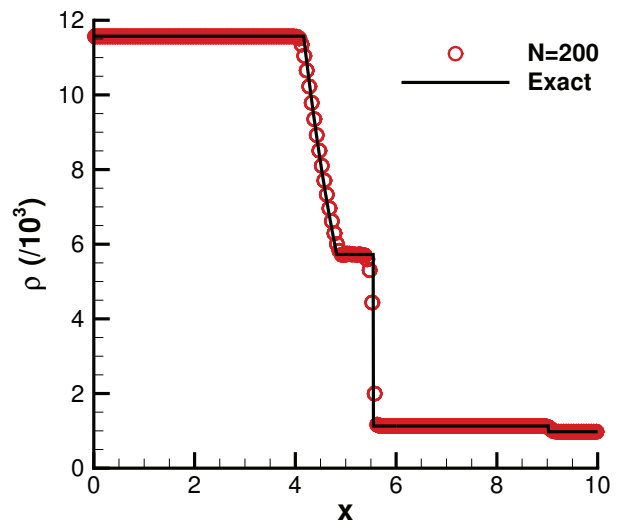

(b)

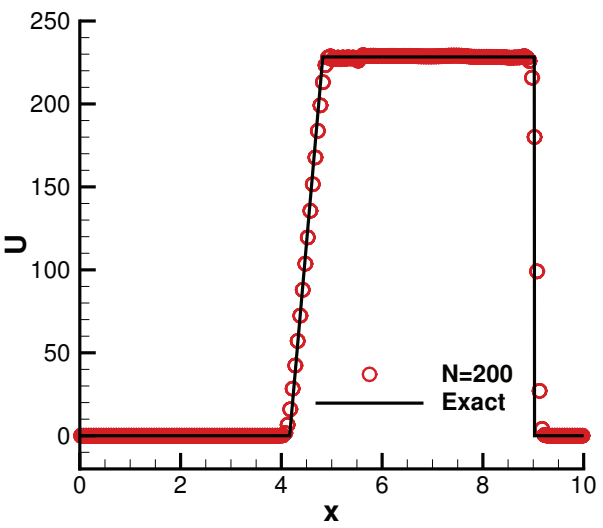

(d)

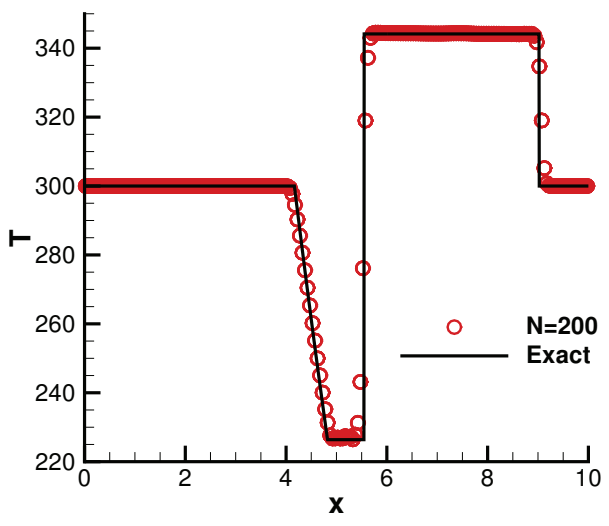

(e)

Figure 4: Profiles of air volume fraction (a), density (b), pressure (c), velocity (d) and temperature (e) for air-water shock tube problem at time $t=2.4 \times 10^{-3}$. 


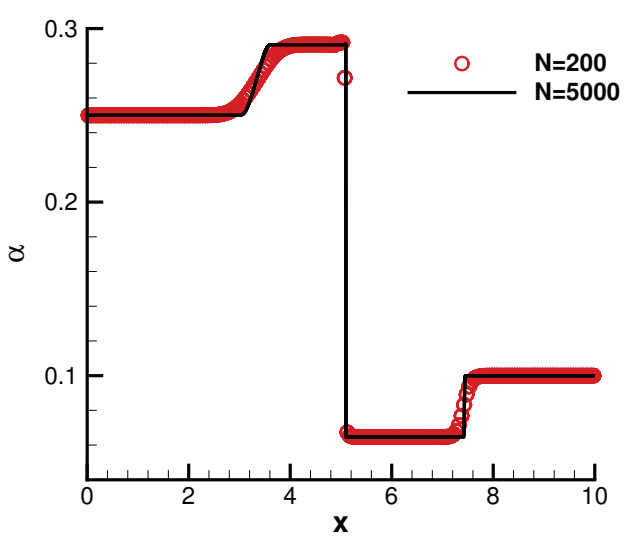

(a)

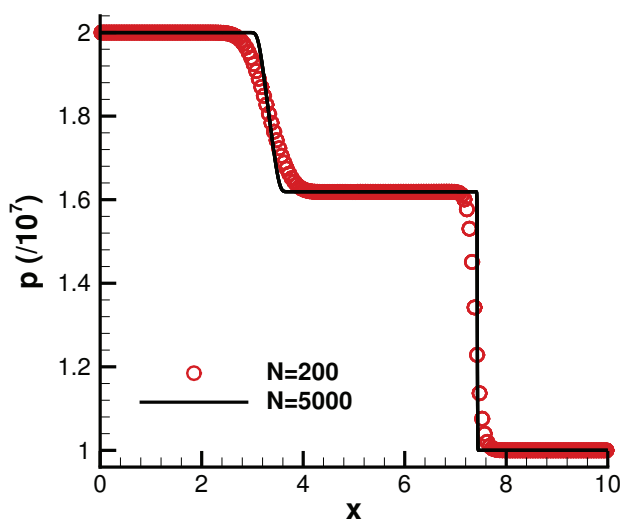

(c)

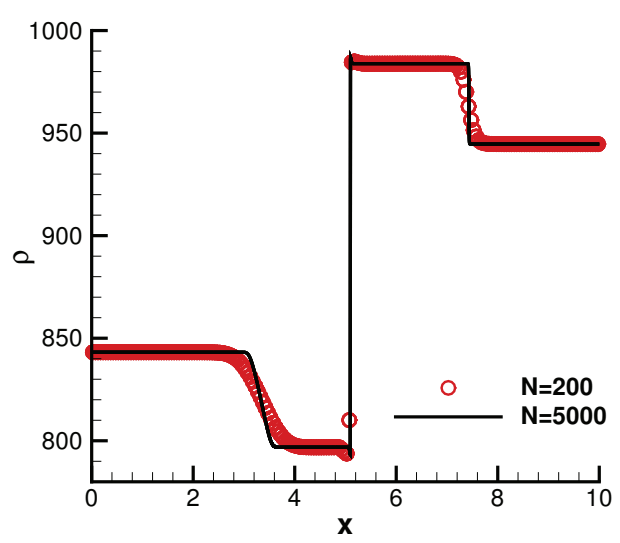

(b)

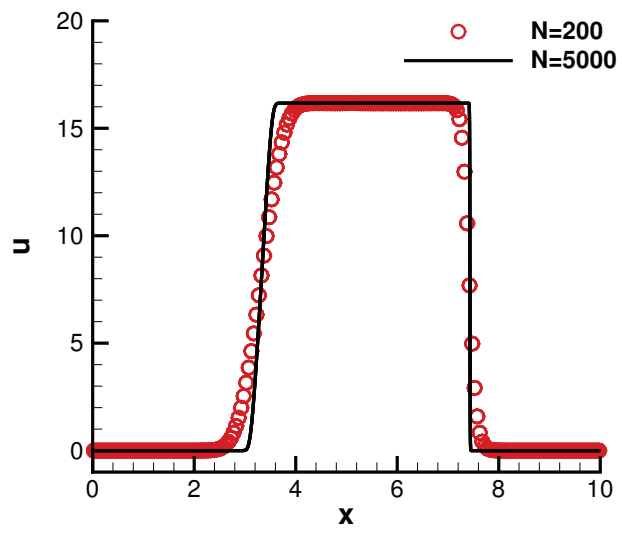

(d)

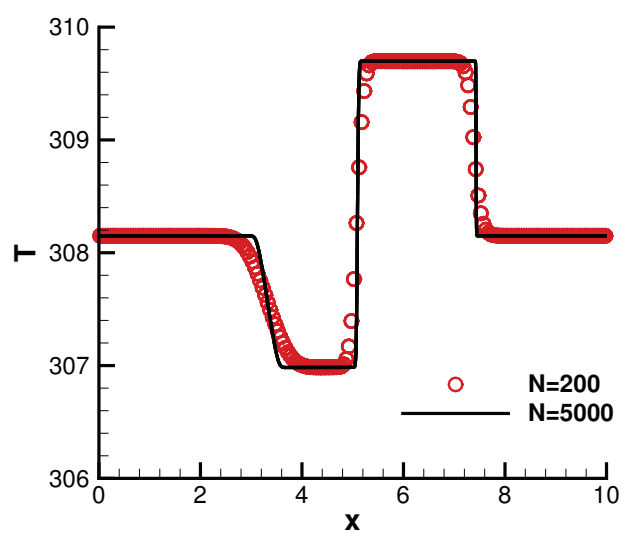

(e)

Figure 5: Profiles of air volume fraction (a), density (b), pressure (c), velocity (d) and temperature (e) for shock tube problem in water-air mixture at time $t=6 \times 10^{-3}$ 
at fluid interfaces [19] and adopting the non-mixing kinetic model but without THINC technique [25]. Again, THINC sharpens the fluid interface effectively, while numerical oscillation can hardly be observed.

It should be noted that GKS-MMF handles the fluid mixing to the equilibrium state only through the projection procedure with different fluxes from cell boundary, which is not included in the flux evaluation. Thus the numerical diffusion seems a bit larger near the rarefaction wave and the shock wave when compared with the results by the mixing kinetic model at fluid interfaces. Of course, here the inviscid assumption is considered. In order to capture the physical mixing, the viscous effect can not be neglected. Then a numerical method for viscous multimaterial flow is required.

\subsection{Shock-water cylinder interaction}

A 2D flow is simulated to further validate the present GKS-MMF. A planar shock wave is traveling with Mach number 3.0 in the stationary air with pressure $p_{i s}=1.0 \times 10^{5} \mathrm{~Pa}$ and density $1.0 \mathrm{~kg} / \mathrm{m}^{3}$, and then hitting a water cylinder with diameter $3.5 \mathrm{~mm}$ [29]. The initial locations for shock wave and cylinder center are $x=8 \mathrm{~mm}$ and $x=10 \mathrm{~mm}$, respectively. The computational domain is chosen as $[0,40 \mathrm{~mm}] \times[0,20 \mathrm{~mm}]$, which is discretized by $1440 \times 600$ rectangular mesh cells and the minimal cell size is $\Delta x=\Delta y=0.01 \mathrm{~mm}$ in the core region, [5mm, $15 \mathrm{~mm}] \times[0,4 \mathrm{~mm}]$. The streamwise length of the domain is long enough to allow the free evolution of flow structures, thus flow variables are set as the initial values on the left and right boundary. The solid wall condition is set on the top boundary and the symmetrical condition is adopted on the bottom boundary due to the symmetry about the horizontal center line. In this test case the convergence criterion to obtain the interface velocity is set as $\hat{\varepsilon}=\max \left(10^{-9} p_{i s}, 10^{-8} \min \left(p^{l}, p^{r}\right)\right)$ to avoid the difficulty near the strong expansion region of water where the pressure is extreme low.

Fig. 6 shows the numerical schlieren photo at different times, which is based on the function $\left(1+(1-\alpha)^{2}\right) \ln (|\nabla \rho|+1)$ to accentuate the weak waves inside water cylinder [29]. The complicated flow structures during the interaction of the moving shock wave and the water cylinder are well captured, including the formation of bow shock before the cylinder (see Fig. 6(a), (b)), the transmitting wave into the water and its primary and secondary reflecting from the rear interface (Fig. 6(c), (d), (e), (f)), along with the formation and disappearance of a low pressure region. In addition, the shock diffraction in the air and the development of complex wave structures behind the cylinder can be clearly observed. These flow characteristics are generally in good agreement with the existing results [29] and the fluid interface is much more sharp when compared with the previous study [25] due to the adoption of directional splitting and THINC techniques.

In Fig. 6(e), (f) small rumples can be observed at the front edge of the water cylinder. They can not be found in the previous results at the same times [25] which shows the good accuracy and resolution of the present improved numerical scheme. As time increases, these rumpled structures grow gradually and even detach from the cylinder, as shown in Fig. 7 at a late stage $t=24 \mu \mathrm{s}$. Although the viscosity and interfacial ten- 


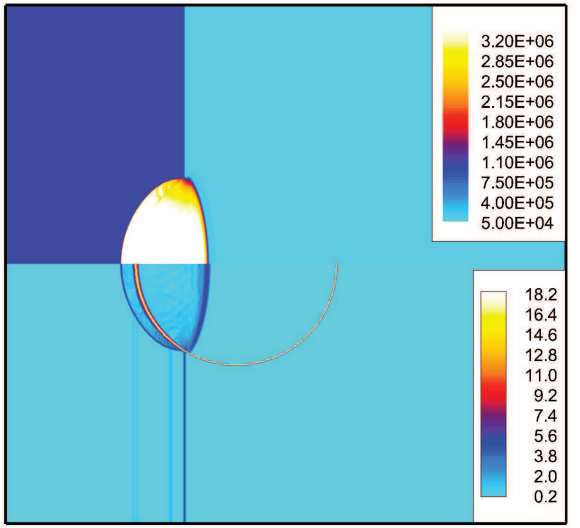

(a)

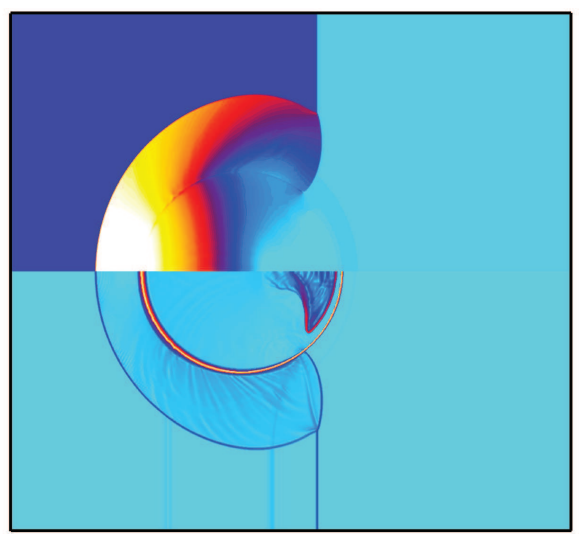

(c)

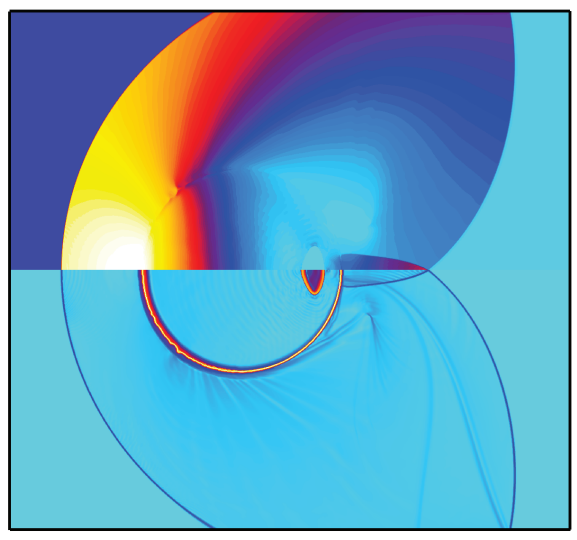

(e)

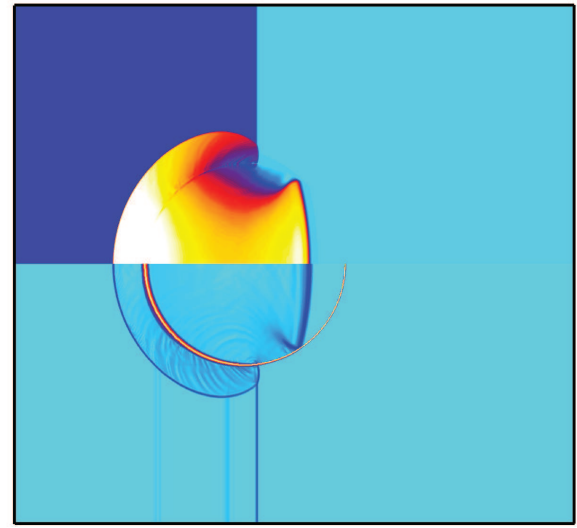

(b)

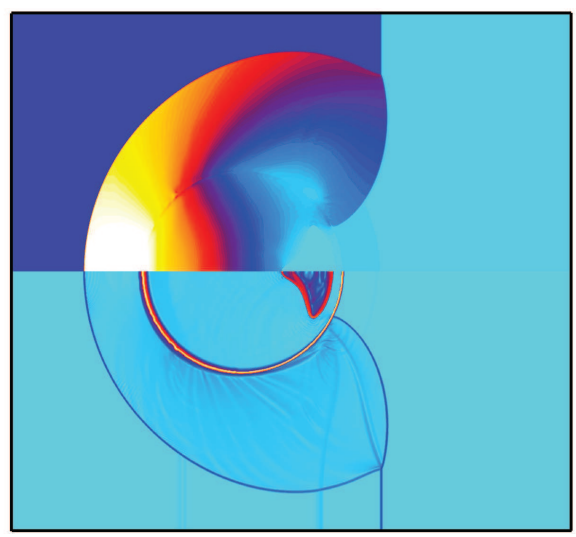

(d)

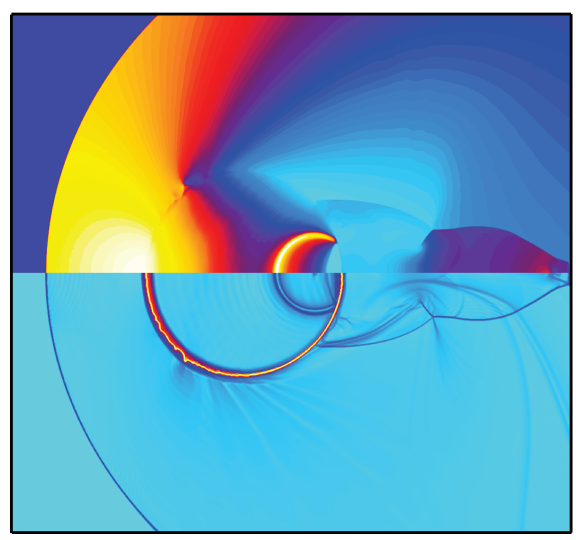

(f)

Figure 6: Instantaneous pressure (upper half) and numerical schlieren photo (lower part) at times $1 \mu \mathrm{s}(\mathrm{a}), 2 \mu \mathrm{s}$

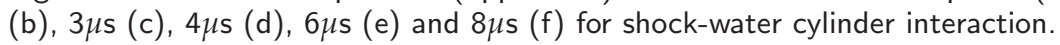




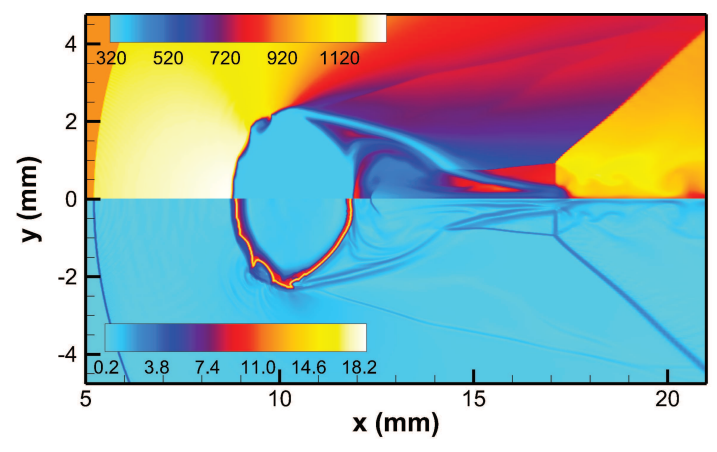

(a)

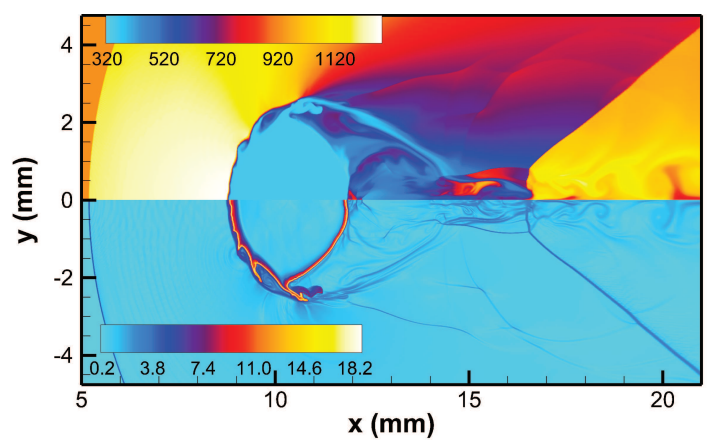

(b)

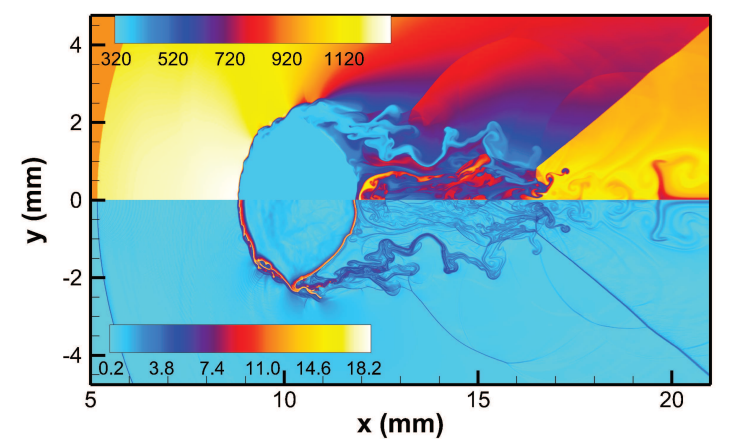

(c)

Figure 7: Instantaneous normalized temperature (upper half) and numerical schlieren photo (lower part) at time $24 \mu \mathrm{s}$ for shock-water cylinder interaction predicted with (a) $720 \times 300$ cells, (b) $1440 \times 600$ cells and (c) $2880 \times 1200$ cells.

sion are not taken into account, the present predicted deformation of the water cylinder and the corresponding flow structures including the front bow shock wave, very complicated waves and vortices in the wake region qualitatively agree with other numerical and experimental studies [2,29,37].

To check the grid effect, two additional computational grids are tried, with $720 \times 300$ cells for the coarse one and $2880 \times 1200$ the fine. Very similar large-scale flow structures are predicted by different grid systems. For small-scale structures it is not surprising that there exists difference such as in the rumples and wake vortices, as the present computation is for inviscid flow. With the fine grid the flattening of water cylinder, the rumpling of fluid interface, the detaching of some water and the entrainment into the air can be clearly captured which are the typical characteristics in the early stage of a cylindrical water drop breakup. As shown in Fig. 8 the predicted shape parameters of the water cylinder by different grid system also show good agreement. Again, due to the rumpling and entrainment near the side edge, more deviation can be observed in these positions.

At last the performance of different iteration method to determine the interface velocity is evaluated in this complicated flow. The initial lower and upper limits for the 


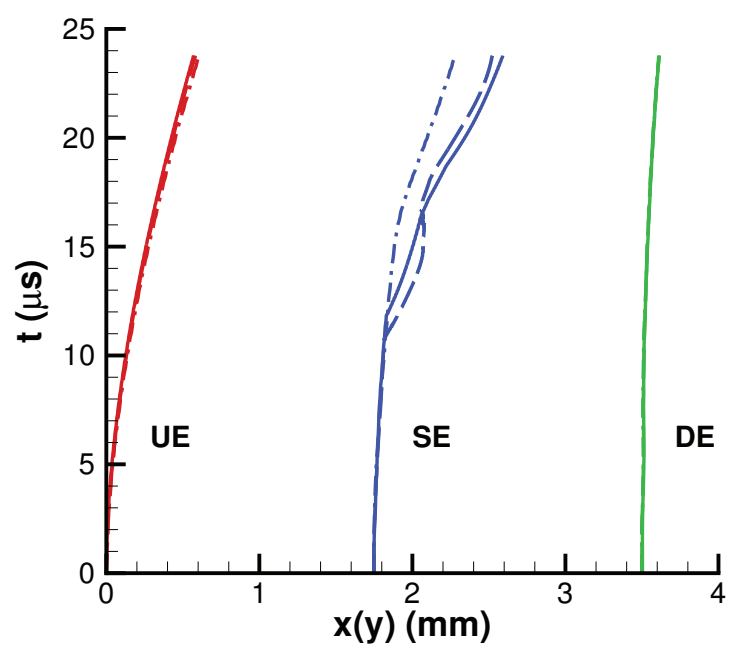

Figure 8: Space-time diagrams of feature points for the shock-water cylinder interaction: UE - upstream edge, DE - downstream edge, SE - side edge of the water cylinder. Dash-dot line represents the results for $720 \times 300$ cells, solid line for $1440 \times 600$ and long dash line for $2880 \times 1200$.

bisection method are given by $U_{\mathrm{int}}^{(0)} \pm 0.1 \max \left(U_{s}^{l}, U_{s}^{r}\right)$ where $U_{s}$ is the sound speed. The number of calls to the function $\mathcal{F}$ in Eq. (3.1) for each method is recorded after a seep of flux computation in the $i$ direction based on the flow filed at time $t=24 \mu \mathrm{s}$. The results are shown in Table 1. When compared with the bisection method, great reduction (nearly in order of magnitude) can be observed by the Steffensen method not only for the average number to compute the force difference, but also for the maximum. The Aitken-Chen method can further reduce the average value but the maximum at some special interfaces looks very large, which means the convergence is not satisfactory and is not good for parallel computing. With the hybrid of these two methods in the present improved Aitken method, the average number shows some decrease and more importantly the maximum is successfully suppressed. Thus the efficiency of GKS-MMF is significantly increased.

Table 1: Numbers of calls to force difference function for different methods to obtain the interface velocity.

\begin{tabular}{||lcccc||}
\hline & Bisection & Steffensen & Aitken-Chen & Improved Aitken \\
\hline Maximum & 43 & 6 & 721 & 7 \\
Average & 36.3 & 4.7 & 4.2 & 4.1 \\
\hline
\end{tabular}

\section{Conclusions}

The recently developed GKS-MMF is improved in two ways. This scheme is based on a non-mixing kinetic Riemann solver for stiffened gases, as well as the HEM model in a cell and the stratified idea to calculate the fluxes at cell interface. An improved Aitken 
method is proposed to obtain the interface velocity in the kinetic Riemann solver, which is a hybrid of the modified Aitken method (Aitken-Chen) and the Steffensen method to reduce the calls to the function. Numerical tests validate the improvement of the present iteration method with significantly less calls to the function not only for the average number but also for the maximum, when compared with existing bisection and Aitken-Chen methods. Considering the computational cost for the force balance function to determine the interface velocity, the reduction of calls is important to the efficiency of the kinetic solver.

The second improvement is the adoption of the THINC reconstruction for the volume fraction and its further hybrid with the van Leer limiter. Compared with existing THINC methods, the present method is very simple under the stratified framework to compute numerical fluxes at a cell interface. It is also capable of resolving fluid interface in mixture. Furthermore, the directional splitting method is used rather than the previous quasi-one-dimensional method in 2D flows. Typical numerical tests, including several water-gas shock tube flows, and the 2D shock-water cylinder interaction flow show that the improved GKS-MMF can capture fluid interfaces much sharper. Meanwhile, the advantages of the original scheme are preserved, including the conservation, robustness and no numerical oscillation at fluid interfaces in the flow with large density ratio and strong shock waves.

The present study shows the strong robustness, high accuracy and efficiency of GKSMMF. To further enhance the scheme, more investigations can be considered, including the combination of moving mesh or adaptive mesh techniques. The expansion to threedimensional or axisymmetric flows is also under consideration.

\section{Acknowledgments}

This work is supported by Science Challenge Project (TZ2016001), National Natural Science Foundation of China (U1430235) and Special Program for Applied Research on Super Computation of the NSFC-Guangdong Joint Fund (the second phase). The author would like to thank the technical support of PARATERA and the "Explorer 100" cluster system of Tsinghua National Laboratory for Information Science and Technology.

\section{References}

[1] R. Abgrall, S. Karni, Computations of compressible multifluids, J. Comput. Phys. 169 (2001) 594-623.

[2] C.-H. Chang, X. Deng, T. G. Theofanous, Direct numerical simulation of interfacial instabilities: A consistent, conservative, all-speed, sharp-interface method, J. Comput. Phys. 242 (2013) 946-990.

[3] S. K. Godunov, A difference method for numerical calculation of discontinuous solutions of the equations of hydrodynamics, Math. Sbornik 47 (3) (1959) 271-306. 
[4] E. F. Toro, Riemann Solvers and Numerical Methods for Fluid Dynamics : A Practical Introduction, 3rd Edition, Springer-Verlag Berlin Heidelberg, Berlin, Heidelberg, 2009.

[5] S. K. Godunov, A. V. Zabrodin, M. I. Ivanov, A. N. Kraiko, G. P. Prokopov, Numerical solution of multidimensional gas dynamics problems, Izdatel'stvo Nauka, Moscow, 1976.

[6] G. Chen, H. Tang, P. Zhang, Second-order accurate Godunov scheme for multicomponent flows on moving triangular meshes, J. Sci. Comput. 34 (1) (2008) 64-86.

[7] M. W. Crochet, K. A. Gonthier, A Riemann problem solution methodology for a class of evolutionary mixture equations with an arbitrary number of components, Appl. Numer. Math. 76 (2014) 145-165.

[8] K. Xu, A kinetic method for hyperbolic-elliptic equations and its application in two-phase flow, J. Comput. Phys. 166 (2) (2001) 383-399.

[9] C. Liu, K. Xu, A unified gas-kinetic scheme for continuum and rarefied flows v: Multiscale and multi-component plasma transport, Commun. Comput. Phys. 22 (2017) 1175-1223.

[10] S. M. Hou, Z. H. Li, X. Y. Jiang, S. Zeng, Numerical study on two-dimensional micro-channel flows using the gas-kinetic unified algorithm, Commun. Comput. Phys. 23 (2018) 1393-1414.

[11] D. Pan, C. Zhong, C. Zhuo, An implicit discrete unified gas-kinetic scheme for simulations of steady flow in all flow regimes, Commun. Comput. Phys. 25 (2019) 1469-1495.

[12] Y. S. Lian, K. Xu, A gas-kinetic scheme for reactive flows, Comput. Fluids 29 (7) (2000) 725748.

[13] S. Jiang, G. X. Ni, A gamma-model BGK scheme for compressible multifluids, Int. J. Numer. Methods Fluids 46 (2) (2004) 163-182.

[14] Q. B. Li, S. Fu, K. Xu, A compressible Navier-Stokes flow solver with scalar transport, J. Comput. Phys. 204 (2) (2005) 692-714.

[15] K. Xu, BGK-based scheme for multicomponent flow calculations, J. Comput. Phys. 134 (1) (1997) 122-133.

[16] A. D. Kotelnikov, D. C. Montgomery, A kinetic method for computing inhomogeneous fluid behavior, J. Comput. Phys. 134 (2) (1997) 364-388.

[17] Y. S. Lian, K. Xu, A gas-kinetic scheme for multimaterial flows and its application in chemical reactions, J. Comput. Phys. 163 (2) (2000) 349-375.

[18] L. Pan, J. X. Cheng, S. H. Wang, K. Xu, A two-stage fourth-order gas-kinetic scheme for compressible multicomponent flows, Commun. Comput. Phys. 23 (2017) 1123-1149.

[19] Q. B. Li, S. Fu, A gas-kinetic BGK scheme for gas-water flow, Comput. Math. Appl. 61 (12) (2011) 3639-3652.

[20] L. Pan, G. P. Zhao, S. H. Wang, A gas-kinetic scheme for the modified Baer-Nunziato model of compressible two-phase flow, Int. J. Numer. Methods Fluids 72 (3) (2013) 320-340.

[21] H.-Z. Tang, H.-M. Wu, Gas-kinetic flux-vector splitting methods for multifluid flow calculation, Comput. Fluid Dyn. J. 41 (5) (2001) 723-734.

[22] Y. Chen, S. Jiang, A non-oscillatory kinetic scheme for multi-component flows with the equation of state for a stiffend gas, J. Comput. Math. 29 (6) (2011) 661-683.

[23] S. Qamar, M. Ahmed, A high order kinetic flux-vector splitting method for the reduced fiveequation model of compressible two-fluid flows, J. Comput. Phys. 228 (2009) 9059-9078.

[24] S. Zia, M. Ahmed, S. Qamar, A gas-kinetic scheme for six-equation two-phase flow model, Applied Mathematics 5 (03) (2014) 453-466.

[25] Q. B. Li, A gas-kinetic Riemann solver for stiffened gas interface and its application in multimaterial flows, Commun. Comput. Phys. 25 (2) (2019) 416-447.

[26] R. Saurel, R. Abgrall, A multiphase Godunov method for compressible multifluid and multiphase flows, J. Comput. Phys. 150 (1999) 425-467. 
[27] R. Abgrall, R. Saurel, Discrete equations for physical and numerical compressible multiphase mixtures, J. Comput. Phys. 186 (2) (2003) 361-396.

[28] H. B. Stewart, B. Wendroff, Two-phase flow: models and methods, J. Comput. Phys. 56 (1984) 363-409.

[29] C.-H. Chang, M.-S. Liou, A robust and accurate approach to computing compressible multiphase flow: Stratified flow model and $\mathrm{AUSM}^{+}$-up scheme, J. Comput. Phys. 225 (1) (2007) 840-873.

[30] F. Xiao, Y. Honma, T. Kono, A simple algebraic interface capturing scheme using hyperbolic tangent function, Int. J. Numer. Methods Fluids 48 (9) (2005) 1023-1040.

[31] K.-M. Shyue, F. Xiao, An Eulerian interface sharpening algorithm for compressible twophase flow: The algebraic THINC approach, J. Comput. Phys. 268 (2014) 326-354.

[32] T. Nonomura, K. Kitamura, K. Fujii, A simple interface sharpening technique with a hyperbolic tangent function applied to compressible two-fluid modeling, J. Comput. Phys. 258 (2014) 95-117.

[33] K. Xu, A gas-kinetic BGK scheme for the Navier-Stokes equations, and its connection with artificial dissipation and Godunov method, J. Comput. Phys. 171 (2001) 289-335.

[34] S.-X. Chen, S.-N. Wang, New linear iterative algorithm and its application in seeking solution of criterion equations of structural optimization, J. Eng. Design 12 (2005) 270-272.

[35] W. D. Gropp, W. Gropp, E. Lusk, A. Skjellum, A. D. F. E. E. Lusk, Using MPI: Portable Parallel Programming with the Message-Passing Interface, Vol. 1, MIT press, 1999.

[36] Y.-Y. Niu, Y.-C. Lin, C.-H. Chang, A further work on multi-phase two-fluid approach for compressible multi-phase flows, Int. J. Numer. Methods Fluids 58 (8) (2008) 879-896.

[37] T. Theofanous, Aerobreakup of Newtonian and viscoelastic liquids, Annu. Rev. Fluid Mech. 43 (2011) 661-690. 